\title{
Ciencia prohibida... ciencia buscada (A propósito de La ciencia del bien y del mal de Javier Echeverría)
}

\author{
VícTOR GÓMEZ PIN
}

Universidad de Barcelona

\begin{abstract}
RESUMEN. Un laberinto era el infinito para Leibniz (quien afirma en varias ocasiones que tanto lo infinitamente grande como lo infinitamente pequeño no son sino ficciones para el pensamiento que busca la explicación de los fenómenos) pero laberinto era asimismo, para él, la cuestión del mal, indisociable de problema del bien. Curtido, desde sus años de joven matemático, en el leibniziano puzzle del infinito y el continuo, Javier Echeverría se confronta ahora al segundo laberinto. Y lo hace restaurando el bíblico relato del árbol cuyo fruto escondería el misterio. Tomando implícitamente una vía opuesta a la que conduce a la kantiana división de la razón, Echeverría apunta a hacer de la polaridad bien-mal objeto de efectiva ciencia. En este escrito discutimos alguna de las implicaciones de su apuesta.
\end{abstract}

Palabras clave: J. Echeverría, pluralismo axiológico, ciencia del bien y del mal.

Muy antigua es la tradición de enfrentarse a los problemas comunes a todos los hombres (es decir, los que con legitimidad pueden ser tildados de filosóficos) apelando a la modalidad de rigor que caracteriza al método geométrico. El nombre de Pitágoras es al respecto emblemático, pero cabría evocar a Leibniz, Spinoza, los discípulos tardíos de Platón y un
AbStRact. A labyrinth was the infinite for Leibniz(who declares on several occasions that,as much from the angle of the infinite large as from that of the infinite small, infinity is nothing but a fiction in the attempts of thought to explain the phenomena). But another labyrinth was also for him the problem of the evilness, together with the correlative problem of goodness. Used, since he was a young mathematician, to the leibnizian puzzle of the infinite, Javier Echeverría faces now the second labyrinth. And he approaches the subject by restoring the biblical myth of the tree that hides the mystery. Taken implicitly the opposite point of view that leads Kant to assert the intrinsic division of the reason, Echeverría, from the very title of his book, aims at goodness and evilness as effective goals for science. In this paper we discuss some implications of Echeverría's bet.

Key words: J. Echeverría, axiological pluralism, goodness and evilness science.

largo etcétera. Javier Echeverría ha dedicado una gran parte de su trayectoria intelectual a explorar e interpretar los textos de Leibniz, en ocasiones manuscritos científicos perdidos entre legajos de la biblioteca de Hannover. Empezaré, pues, esta reflexión a la que da pie su último libro (La ciencia del bien y del mal, Herder, Barcelona, 2007), evocando un 
párrafo sobre el que Francisco Fernández (leibniziano por él formado en la vieja facultad de filosofía de Zorroaga) se complacía en meditar:

«Hay dos célebres laberintos en los que a menudo la razón se extravía: el primero concierne a la importantísima cuestión de la libertad y la necesidad, especialmente en lo relativo a la producción y el origen del mal; el segundo consiste en la discusión de la continuidad y de los indivisibles, que parecen constituir los elementos del continuo, asunto en el que entra en juego la consideración del infinito: el primer laberinto ocupa a todo el género humano; el segundo afecta tan sólo a los filósofos».

Quizás Leibniz se equivoca al afirmar que el problema del infinito y el continuo sólo afecta a los filósofos, pues al decir de Hilbert en el mismo se halla en juego ni más ni menos que «la dignidad misma del espíritu humano». En cualquier caso, curtido en el problema del continuo, Echeverría se enfrenta ahora al problema del mal, y lo hace evocando uno de los relatos mayormente configuradotes de nuestra civilización: la serpiente doblega la prudencia de nuestros primeros ancestros con la promesa de plenitud (equiparación a los dioses) que resultaría de consumir el fruto de un árbol ubicado, junto al árbol de la vida, en el centro del Paraíso; la mujer es la primera en caer y su pusilánime esposo la sigue.

Sabido es que el árbol del Bien y del Mal no depara a los infractores de la prohibición divina otra lucidez que el de la conciencia de su desamparo: «entonces se les abrieron a entrambos los ojos, se dieron cuenta de que estaban desnudos, y cogiendo hojas de higuera se hicieron unos ceñidores». Javier Echeverría indica que, como resultado de tal recubrimiento de la desnudez, los meros simios que eran Adán y Eva abandonaron la condición meramente animal. La pérdida de la relación inmediata con la naturaleza sería consecuencia de caer en ese estado de estupor que constituye el percibirse como ser que ignora.

Así pues, la manzana encerraba una promesa de saber y la moraleja del castigo y la vergüenza viene a indicar que $s a$ ber, e incluso aspirar a ello, es lo que está esencialmente prohibido («si quieres ser feliz, como me dices...»). Mas Aristóteles, como tantos otros de los grandes del pensamiento y del verbo, erige la exigencia de saber en marca distintiva de nuestra condición. De ahí lo pertinente de recordar con Echeverría que Eva representa el primer arquetipo de quien «prefirió el conocimiento a la sumisión», o sea, del filósofo.

Sobre la aspiración al saber no ha dejado nunca de pesar la amenaza del castigo, siendo el destino de Sócrates paradigma de que la lucha por ejercer el juicio, es decir, por dejar de vivir en conformidad a prejuicios, puede ser intolerable para el orden social establecido. Ciertamente, desde el origen de la humanidad ha habido enormes avances en el conocimiento, mas tal progreso se ha ido configurando en base a una distinción radical cuyo no respeto hace interesantísimo el libro que aquí glosamos. El físico Erwing Schrödinger señalaba que un momento determinante de nuestra relación con el orden cósmico es la introducción de una suerte de postulado que hace de la naturaleza algo intrínsicamente reductible a la razón. Una gran civilización puede sustentarse en respetar la naturaleza o divinizarla, pero algo cambia radicalmente cuando se introduce la premisa de que esa naturaleza es cognoscible, objetivizable, objeto de ciencia, es decir, de una disposición del espíritu que no es, de entrada, considerada apta para la expresión de los fenómenos a los que se alude precisamente mediante expresiones como bien o mal.

Se computa, describe y prevé el comportamiento del átomo de hidrógeno y 
hasta (muy recientemente) el de un fotón cercenado en una cavidad, se computa y describe un sistema tan complejo como es el genoma del chimpancé, o el del ser humano..., pero se desespera de llegar a describir, computar y hacer previsiones respecto del conjunto de variables que permitirían emitir un juicio apodíctico sobre lo moralmente fundado de la decisión de Tony Blair de comprometer a su país en el pantano iraquí. Cabría, en suma, una ciencia de la naturaleza, pero no cabría una ciencia del bien y del mal.

Heidegger ha enfatizado el hecho de lo que supone la subordinación de nuestra relación con el ente a la ratio, entendida como el conjunto unificado de condiciones a priori que posibilitan la objetivización del orden natural. Y aunque, como señala el propio Heidegger, Kant también sitúa en la ratio las condiciones posibilitadoras de la libertad, aunque quepa barruntar que se da una razón común, la frontera que separa ambos registros parece irreductible. Kant sugiere que en el dominio práctico la razón invierte, por decirlo así, más si cabe que en el dominio especulativo. Pues en el conocimiento, se daría la paradoja de que la razón misma dejaría el papel principal a una facultad inferior, a saber, el entendimiento. En la perspectiva kantiana, tal sacrificio de la razón cuando de conocer se trata, no hace más que poner de relieve su dignidad esencial, su plena actualización, en el dominio práctico. Determinado mi espíritu por el puro amor a la ley «sin intromisión de otras influencias de mi sensibilidad»; activada la facultad de desear sin mediación de una representación de objeto (ya sea trascendental o empírico); operativas las ideas (Dios, Mundo, Alma) que tratándose de conocimiento sólo jugaban un papel regulador (aplicación de una categoría de la relación a los objetos cognoscibles), entonces no prima el conocimiento, prima el interés superior de la ra- zón. ¿Satisfactoria para todo el mundo esta perspectiva que escinde razón práctica y razón cognoscitiva? ¿Hemos, en materia de ética, de renunciar a conocer? Algunos resisten:

Javier Echeverría va directamente al asunto preguntándose en el capítulo dos de su libro»: «¿Es posible una ciencia del bien y del mal?». La respuesta a su juicio es afirmativa, aunque ampliando suficientemente el concepto de ciencia y dialectizando lo que cabe entender por bien y por mal. Tesis central respecto a esto último es no sólo la intrínseca polaridad de ambos conceptos (no cabe bien sin mal, ni viceversa) sino asimismo el carácter mutable y relativo de lo que cabe experimentar (y no sólo entender) en relación al bien y al mal. Por citar un ejemplo, ampliamente tratado en uno de los capítulos del libro: como resultado de la transmutación de valores que supuso la Reforma, y de las nuevas condiciones sociales, la pobreza, la mendicidad y el vagabundeo, «en lugar de ser condiciones naturales debidas al designio divino, comenzaron a ser interpretadas como defectos y vicios, si no como delitos».

En cuanto a la ciencia, Javier Echeverría rechaza una concepción monista de la misma, al igual que rechaza «monoteísmo, monarquía, monocausalismo, monocentrismo, identidad única, etc.». De ahí que su disposición de espíritu sea la de servirse de una metodología en la que el rigor no sea óbice para la pluralidad de perspectivas.

Así la «ciencia del bien y del mal» que este libro nos depara tiene uno de sus pilares en el método filosófico, que parte de los conceptos operativos en el lenguaje ordinario. Ello se complementa con una metodología empírica, que engloba experimentos mentales relativos a la vivencia del bien y del mal por parte de los animales no humanos (en cuyo lugar nos invita a situarnos) y con la asunción de 
una serie de teorías científicas, Darwinismo en primer lugar, pero también, por ejemplo, la teoría general de sistemas.

Nuclear en el libro es, sin embargo, la última parte, por la que el autor nos invita a empezar la lectura y que consiste ni más ni menos que en una ciencia del bien y del mal expuesta more geometrico. Todos los conceptos que operan en el libro son aquí analizados, justificados y, sobre todo, fertilizados, configurando definiciones, axiomas, postulados, teoremas, corolarios, escolios, refutaciones, respuestas..., en suma: todo aquello que de forma arquetípica, desde Euclides cuando menos, se articula como texto científico.

El autor indica que su exposición more geometrico será posiblemente la parte del libro vivida por el lector como más problemática y hasta «intempestiva». No lo creo así. Su exposición axiomática no trasciende el lenguaje natural y no tiene en consecuencia que ser tomada más que como apreciable esfuerzo por alcanzar la «claridad y distinción» en el tratamiento del problema, lo cual es siempre de agradecer en un texto filosófico. Otra cosa sería la pretensión de que el alfabeto oculto, tratándose de cuestiones morales, fuera tan matemático como el que se revela operativo en la ciencia de la naturaleza. Personalmente he seguido el consejo dado por el autor, empezando el libro por esa parte y creo que se consigue en ella un efectivo equilibrio entre el rigor lógico y la apuesta por no excluir al lector no filósofo de un problema que a todos nos concierne La cuestión esencial es si el bien y el mal son o no objeto de saber. Que la modalidad de rigor coincida eventualmente con la modalidad de rigor propia de la exposición geométrica es ya relativamente secundario, salvo que se postule que esta última tiene el monopolio de la legitimidad en materia de conocimiento. Pero en este caso, la tendencia suele ser el pedir el sacrificio del lengua- je natural, no sólo intrínsecamente equívoco, sino fértil precisamente en razón de esa equivocidad. Me consta que en materia de escritura Echeverría es obsesivamente escrupuloso (una de sus exigencias es que se juzguen los textos estrictamente matemáticos también por el estilo) $\mathrm{y}$, emulando a Spinoza, tiene todo el derecho del mundo a exponer los contenidos del saber (siempre en base al postulado de que tal saber es posible) con método geométrico. Lo eventualmente problemático de esta ambiciosa apuesta va por otro lado, al que luego me referiré, pues el libro va mucho más allá de esa tercera parte concebida more geometrico.

Los «comentarios y debates» de la segunda parte consiguen el objetivo de incitar efectivamente al lector no tanto a asentir como a comentar y debatir. Y, desde luego, lo más excitante del libro (literariamente incluso) reside en los «experimentos» que constituyen la primera parte, en los que se despliega la vena literaria que siempre ha animado a este filósofo, y su interés por las situaciones en las que (en términos kantianos) es la imaginación y no el entendimiento la facultad que legisla. Baste contemplar los títulos de algunos de estos singulares «experimentos mentales»: seamos plantas, seamos algas, seamos lobos, y sobre todo seamos serpientes, perspectiva que es explícitamente aquélla desde la que se concibe el libro.

Hay una noción en el libro que presenta un elevado interés, la de Racionalidad axiológica acotada, elaborada en colaboración con Francisco Álvarez, sobre la cual se anuncia un próximo libro. En síntesis: «A) la racionalidad depende de los valores que guían las acciones, incluidas las acciones mentales». «B) la racionalidad es limitada, puesto que todo valor tiene cotas máximas de satisfacción en cada circunstancia, por encima de las cuales deviene un contravalor». Echeverría 
señala que lo apuntado en A permitiría superar la distinción kantiana entre razón pura cognoscitiva y razón práctica. Ello se efectuaría, en principio, a favor de la segunda, puesto que los instrumentos de la primera (conceptos, definiciones, hipótesis, leyes, etc.), serían resultado de que las máximas subjetivas de acción vendrían determinadas por valores epistémicos, un tipo de valores todo lo especial que se quiera, pero valores al fin y al cabo. Para apuntalar su concepción, Echeverría hace un repaso de las teorías clásicas de la racionalidad: la leibniziana; el racionalismo crítico de Popper; la teoría de la decisión racional, surgida de la teoría económica y vinculada a la racionalidad instrumental (de la cual nos dice que se ha convertido en un auténtico canon de la racionalidad, yo añadiría que desgraciadamente).

Pues bien, mi empatía es total con la conjetura de que el bagaje posibilitador del conocimiento es resultado de la operatividad de una clase especial de valores, aunque parta de horizontes teóricos diferentes de los de Echeverría, extraiga de la conjetura otros corolarios y quizás me posicione diferentemente a la hora de determinar si el conjunto de proposiciones que forjan un discurso (científico o no) del bien y del mal, remite, en última instancia, a alguna auténtica noción común, es decir, a algo que en todos y cada uno de nosotros tendría la dignidad de lo incondicionado e incuestionable. Obviamente estoy refiriéndome de nuevo (aunque considerando otra vertiente) a la tesis kantiana, según la cual el ser de razón se halla permanentemente sometido al imperativo consistente en no negar, en circunstancia alguna, la primacía de la razón misma. Imperativo del que se desprende como mero corolario que no debe jamás instrumentalizarse o subordinar a los demás seres de razón, es decir, el respeto a la persona (algunos, absurdamente, precisan persona humana) que ha sido clásicamente considerado como expresión cabal del comportamiento ético. Vamos por partes.

El asunto de la pertinencia o no pertinencia de abordar la cuestión ética con las armas propias del conocimiento se vincula a un debate obsesivo en nuestro tiempo y que remite, al fin y al cabo, a determinar si hay o no una ciencia del hombre. Cabe considerar que el ser humano es una especie natural más, es decir, sin otras singularidades que las que caracterizan a cualquier especie animal frente a las que difieren de ella; en tal caso sería posible una ciencia del hombre (incluido su lenguaje y su mente), como es posible, potencialmente al menos, una ciencia de cualquier otra entidad natural. Cabe, por el contrario, considerar que el hombre supone una radical ruptura de continuidad en el registro evolutivo, ruptura que lo hace difícilmente reductible a la objetivación exigida por la ciencia natural. Sentadas así las cosas, la discusión podría prolongarse al infinito. Mas los partidarios de la primera posición dan un paso radical cuando postulan que sólo el saber vehiculado por la ciencia es apto a sustentar en base auténticamente racional otros discursos, concretamente el discurso ético.

Caricaturizando apenas, los más dogmáticos de los que mantienen tales posiciones vienen a decir: nuestro discurso sobre los animales, los humanos, y la relación entre ellos se sostiene desde la certeza científica, mientras que toda ética de inspiración kantiana (que sostiene la irreductible dignidad del ser humano) se fundamenta en prejuicios ideológicos o religiosos; ética de gente precientífica, cuando no ética de mal intencionados que - por ejemplo - se aferran a prejuicios respecto al lazo hombre-animal porque tal cosa les conviene. Pues bien, cabe elevarse contra esta presentación enarbolando la hipótesis contraria (empática con la 
tesis de Echeverría sobre el peso que tendrían los valores también en el registro epistémico): la ciencia misma sería un resultado de la singularísima disposición que se da en el ser humano (y sólo en el ser humano, enfatizo por mi parte) que cabe tildar de ética, a saber, la subordinación de los lazos con el entorno natural, con los demás humanos y hasta con uno mismo a exigencias que no se hallan determinadas por la darwiniana lucha por la subsistencia. Obviamente para ver en la ciencia un corolario de la disposición ética, ha de entenderse por ciencia lo que reivindicaba René Thom (Medalla Fields de Matemáticas y lector en la lengua original de los textos de Aristóteles), a saber, una tarea motivada exclusivamente por exigencias de inteligibilidad, exigencias de enriquecer el lenguaje y la mente; tarea de la que, por añadidura, pero sólo por añadidura, cabría esperar resultados desde el punto de vista de los intereses relativos a la subsistencia y al ornato de la vida. El ser humano, en ocasiones, asumiría plenamente su condición de ser racional y si en tal asunción (de la que se infiere inmediatamente la exigencia de no instrumentalización de los seres de razón) se barrunta la esencia de la ética, y la matriz del deseo de hacer el mundo inteligible, entonces la ética no puede ser una parte entre otras del corpus del saber actualizado. La ciencia, en suma, no sería sino una de las modalidades en las que se refleja la irremediable distanciación respecto a la inmediatez del orden natural que supone la emergencia del orden simbólico. Distanciación que entre, otras cosas justifica que Echeverría pueda escribir, bajo la rúbrica algo provocativa de una calidad de vida post mortem, lo siguiente:

«Los bienes y los males van más allá de la muerte y, por ende, del gozo y del sufrimiento. Después de muertos muchos seres humanos no dejan de existir. Tal es el caso de los personajes que pasan a la historia. No existen física ni corporalmente, pero sí socialmente. Los seres humanos mantenemos la existencia virtual de multitud de personas, animales y cosas que han dejado de estar presentes físicamente desde siglos, e incluso que nunca existieron corporalmente. Es una de nuestras características como animales sociales, sin $p a-$ rangón con otras especies (la cursiva es mía). La cuestión de bien y del mal también se plantea en la esfera de la memoria».

Sí, la capacidad de conferir entidad más allá de la muerte es exclusiva de los seres de razón, y por ello mismo el bien y el mal experimentados por los humanos no pueden ser reducidos al inmediato sentir de los demás animales. De ahí que el antropocentrismo en estas cuestiones no pueda, como algunos pretenden, ser despachado de un plumazo. No es, desde luego, el caso de Echeverría, pero creo que en alguna ocasión no es totalmente consecuente con el párrafo de su libro que acabo de citar. Así cuando describe modelos de acción basados en un único sistema de valores escribe: «Sacrificar la vida por el hormiguero, por la colmena, o por las crías, aunque el sacrificio se haga tan sólo por instinto..., caracteriza la primacía de un tipo de valores sobre todos los demás» Pues bien, nunca es obvio enfatizar que aquello que constituye un valor meramente para una facultad instintiva pertenece a un registro diferente a lo valorable por un instinto intrínsicamente mediatizado por la razón. Ello aun dejando de lado las circunstancias en las que el ser humano responde a motivos que ya nada tienen que ver con el instinto, y que incluso entran en contradicción con el mismo Kant una vez más..., que cuando creías haberlo superado te está esperando en la esquina (cosa que Foucault decía de Hegel y que pasa con tantos otros «perros muertos» de la filosofía).

El formalismo kantiano en materia de ética, sustentado en el imperativo categórico (que jugaría en el registro de la 
moral un papel análogo al del principio de no contradicción en el plano cognoscitivo) ha sido objeto de toda clase de críticas. El marxismo venía ya a recordar que la cuestión de la realización plena de la naturaleza racional del hombre (comportamientos éticos incluidos) pasa en primera instancia por alcanzar las condiciones materiales de la misma. Pero el movimiento social que empujaba a esta actitud y le confería fuerza ha sido vencido, de tal manera que apelar hoy a objetivos de global emancipación (lo que hoy se entiende por global casa más bien con genuflexión) es exponerse cuando menos al sarcasmo.

Resignados a una situación en la que no caben más libertades que las meramente formales, parece que el formalismo kantiano en materia de moral podría ser fácilmente recuperado. Si no es el caso, si de alguna manera Kant se ha convertido en el enemigo primordial de algunas de las ideologías contemporáneas de mayor peso, es porque sigue habiendo en sus planteamientos algo radicalmente subversivo. Pues la imagen de seres racionales condenados a la alternancia entre tareas sociales embrutecedoras y escapatorias lúdicas, o afectivas, que son a la vez complemento y coartada de las anteriores (esa vida, en suma que antes se designaba mediante el término alienación), no deja de ser permanente afrenta para todo aquel que sienta su racionalidad como singularidad absoluta en el seno de lo viviente. De ahí que, en materia de ética, se abra camino una actitud que empieza por negar la mayor del kantismo, postulando que el único fundamento de la eticidad es la vivencia de la comunidad de destino con los seres meramente vivos, la empatía con los seres meramente susceptibles de experimentar dolor y placer.
Javier Echeverría tiene en su reflexión que habérselas con los evocados toros... y alguno más. En su honrado esfuerzo por tomar de la tradición filosófica meramente lo que sirve, efectúa una crítica de Aristóteles en lo referente a la pertinencia de sus procedimientos de clasificación (organización de los tipos de bienes y males en géneros y especies), pero recupera su concepto fundamental de Frónesis, prudencia. La prudencia es de rigor, en base a la convicción de que los bienes y los males cambian según las circunstancias. «La axiología ha de ser dinámica y situacional, no estática e intemporal»», escribe, rechazando, sin embargo la caída en el relativismo, a fortiori en el escepticismo. Pues bien, no estoy seguro de que tal compromiso sea viable.

No expreso tanto el resultado de un razonamiento como un sentimiento inmediato, al atreverme a decir que, en materia de bien y de mal, hay en última instancia una alternativa absoluta, lo que no equivale a decir que estén bajo control las variables que determinan el comportamiento objetivo. Efectivamente, la prudencia implica grados y los animales - a algunos de los cuales Aristóteles atribuye tal virtud-dosifican su comportamiento tanto al acechar o atacar como al amagar o huir. Pero además de prudencia se espera del hombre entereza, disposición para la cual Aristóteles tenía también un término, andreia, etimológicamente hombría pero que atribuía tanto a hombres como a mujeres. Determinar si, en última instancia, cuando lo esencial se juega, la disposición ética sigue siendo conciliable con la prudencia, determinar hasta dónde esta última acompaña a la entereza, es la tarea pendiente en este esfuerzo de Javier Echeverría en pos de una ciencia del bien y del mal. 